01,11

\title{
Электронная структура и магнитный фазовый переход в геликоидальных ферромагнетиках $\mathrm{Fe}_{1-x} \mathrm{Co}_{x} \mathrm{Si}$
}

\author{
(C) А.А. Повзнер, А.Г. Волков, Т.А. Ноговицына \\ Уральский федеральный университет им. Б.Н. Ельцина, \\ Екатеринбург, Россия \\ E-mail: a.a.povzner@urfu.ru
}

(Поступила в Редакцию 10 июля 2017 г.)

$\mathrm{LSDA}+\mathrm{U}+\mathrm{SO}$-расчеты электронной структуры геликоидальных ферромагнетиков $\mathrm{Fe}_{1-x} \mathrm{Co}_{x} \mathrm{Si}$ в приближении виртуального кристалла, дополнены учетом взаимодействия Дзялошинского-Мория и ферромагнитных флуктуаций спиновой плотности коллективизированных $d$-электронов с хаббардовскими взаимодействиями на хаотически распределенных по узлам атомах $\mathrm{Fe}$ и Co. Уравнение магнитного состояния в развитой модели описывает геликоидальный ферромагнетизм и его исчезновение, сопровождаемое возникновением максимума однородной магнитной восприимчивости при температуре $T_{C}$ и киральных флуктуаций локальной намагниченности при $T>T_{C}$. Исследуются причины того, почему магнитный вклад в теплоемкость при фазовом магнитном переходе изменяется монотонно, а объемный коэффициент теплового расширения (ОКТР) в области низких температур отрицателен и имеет широкий минимум вблизи $T_{C}$. Показано, что ОКТР при переходе в парамагнитное состояние (при температуре $T_{S}$ ), меняет знак.

Результаты были получены в рамках задания Министерства образования и науки РФ (контракт 3.9521.2017/8.9).

DOI: $10.21883 /$ FTT.2018.02.45372.232

\section{1. Введение}

Кристаллическая структура сильно коррелированных геликоидальных ферромагнетиков на основе соединений и сплавов $\mathrm{MnSi}, \mathrm{Fe}_{1-x} \mathrm{Co}_{x} \mathrm{Si}$ и др. относится к структурному типу В20 с пространственной группой $P 2{ }_{1} 3$, для которой характерно отсутствие центра инверсии. Такая симметрия приводит к антисимметричному релятивистскому обмену Дзялошинского-Мория (ДМ) [1]. В соответствии с моделью Бака-Янсена [2], конкуренция DМ-взаимодействия с неоднородным обменным взаимодействием в ферромагнетике приводит к наблюдаемому на эксперименте геликоидальному ферромагнетизму с аномально большими периодами спиновой сверхструктуры [3].

Эксперимент показывает, что при температуре $T_{C}$ в рассматриваемых киральных магнетиках наблюдается магнитный фазовый переход, приводящий к исчезновению геликоидального ферромагнитного упорядочения („катастрофа“ модели Бака-Янсена). Этот переход сопровождается формированием температурных максимумов однородной магнитной восприимчивости [4]. При этом картина температурных зависимостей теплоемкости и ОКТР не однозначна. Так в $\mathrm{MnSi}$ вблизи $\mathrm{T}_{C}$ возникают лямбда-подобные аномалии, с последующим формированием „плеча“ на температурных зависимостях теплоемкости и ОКТР [5]. В геликоидальных ферромагнетиках $\mathrm{Fe}_{1-x} \mathrm{Co}_{x} \mathrm{Si}$ вблизи температуры максимума однородной магнитной восприимчивости $\left(T_{C}\right)$ экспериментально обнаружен сильно размытый по температуре минимум отрицательного ОКТР, но какие-либо особен- ности на температурной зависимости теплоемкости не наблюдаются [6].

Согласно результатам исследований малоуглового рассеяния поляризованных нейтронов выше температуры $T_{C}$ в рассматриваемых геликоидальных ферромагнетиках сохраняется спиновый ближний порядок с киральными флуктуациями [7,8]. Однако природа и причины возникновения такого спинового ближнего порядка, температурные границы его существования, связь с параметрами электронной и атомной структуры не установлены. Во внешнем магнитном поле в области киральных флуктуаций возникает скирмионная фаза, границы существования которой зависят от их химического состава $\left(\mathrm{MnSi}, \mathrm{Fe}_{1-x} \mathrm{Co}_{x} \mathrm{Si}, \mathrm{FeGe}\right.$ и др.) $[4,9,10]$.

$A b$ initio $\mathrm{LSDA}+\mathrm{U}+\mathrm{SO}$ - расчеты электронных спектров для $\mathrm{MnSi}, \quad \mathrm{Fe}_{0.5} \mathrm{Co}_{0.5} \mathrm{Si}, \quad \mathrm{Fe}_{0.2} \mathrm{Co}_{0.8} \mathrm{Si}$ и $\mathrm{Fe}_{0.8} \mathrm{Co}_{0.2} \mathrm{Si}$ с кристаллической структурой В20 описывают возникновение в непосредственной энергетической окрестности химического потенциала разделенных энергетической щелью $d$-подзон. Нижняя по энергии зона содержит смесь триплетных и синглетных $e_{g}-$ и $t_{2 g}$-состояний, а верхняя зона формируется преимущественно синглетными $t_{0}$-электронными состояниями [11]. $\mathrm{B} \mathrm{MnSi}$ химический потенциал расположен в нижней зоне [12], а в геликоидальных ферромагнетиках $\mathrm{Fe}_{1-x} \mathrm{Co}_{x} \mathrm{Si}$ в верхней зоне [11]. При этом рассчитанные в рамках LSDA + U-моделирования значения магнитных моментов, в обоих случаях заметно превышают значения, получаемые на эксперименте $[11,12]$. Ранее [13] было показано, что в моносилициде марганца возникают достаточно большие по амплитуде квантовые ну- 
левые спиновые флуктуации, учет которых позволяет согласовать LSDA-расчеты магнитных моментов с их экспериментально наблюдаемыми значениями. При этом нулевые спиновые флуктуации скачкообразно исчезают при магнитном фазовом переходе первого рода [13], что приводит к скачку объема и лямбда-подобным аномалиям теплоемкости и ОКТР. В киральных ферромагнетиках на основе $\mathrm{Fe}_{1-x} \mathrm{Co}_{x} \mathrm{Si}$, возникающие при заполнении синглетных $t_{0}$ состояний магнитные моменты и их флуктуации, пока не изучены.

\section{2. Модель}

Рассмотрим сильно коррелированную электронную систему $t_{0}$-зоны киральных магнетиков $\mathrm{Fe}_{1-x} \mathrm{Co}_{x} \mathrm{Si}$ c гамильтонианом

$$
H=H_{0}+\delta H_{U}+H_{\mathrm{DM}}
$$

Здесь $H_{0}=\sum_{\mathbf{k}, \sigma} \varepsilon_{\mathbf{k}, \sigma}^{(\mathrm{LSDA})} a_{\mathbf{k}, \sigma}^{+} a_{\mathbf{k}, \sigma}-$ гамильтониан $d$-электронов в $t_{0}$-орбитальном состоянии, $a_{\mathbf{k}, \sigma}^{+}\left(a_{\mathbf{k}, \sigma}\right)-$ оператор рождения (уничтожения) электрона в $t_{0}$-зоне, $\mathbf{k}$ - вектор квазиимпульса, $\sigma(= \pm 1)$ - спиновый индекс, $\varepsilon_{\mathbf{k}, \sigma}^{(\mathrm{LSDA})}=\varepsilon_{\mathbf{k}}^{(\mathrm{LDA})}-\sigma \Delta-$ электронный спектр $d$-электронов в $t_{0}$-орбитальном состоянии, рассчитанный в LSDA + U + SO-приближении и приближении виртуального кристалла [11], и отсчитываемый от энергии химического потенциала рассматриваемой электронной системы,

$$
\begin{aligned}
& \delta H_{U}=\left(U_{\mathrm{Fe}}-U_{\mathrm{Co}}\right) \\
& \quad \times \sum_{\boldsymbol{v}} \delta p_{\boldsymbol{v}}\left|\sum_{\sigma} \sigma\left\langle n_{\sigma}\right\rangle_{0} \delta S_{\boldsymbol{v}}^{(z)}-\sum_{\sigma}\left\langle n_{\sigma}\right\rangle_{0} \delta n_{\boldsymbol{v}} / 2\right| \\
& \quad-\sum_{\boldsymbol{v}}\left(U_{\mathrm{Fe}}\left(1-p_{\boldsymbol{v}}\right)+U_{\mathrm{Co}} p_{\boldsymbol{v}}\right)\left[\left(\delta S_{\boldsymbol{v}}^{(z)}\right)-\left(\delta n_{\boldsymbol{v}}\right)^{2} / 4\right]
\end{aligned}
$$

поправка, учитывающая флуктуации электронной плотности, обусловленные межэлектронными корреляциями и различием параметров хаббардовского взаимодействия на узлах оккупированных атомами кобальта или железа $\left(U_{\mathrm{Co}}\right.$ и $U_{\mathrm{Fe}}-$ соответственно) $[14], \delta p_{\boldsymbol{v}}=p_{\boldsymbol{v}}-p, p-$ концентрация атомов кобальта, $p_{v}-$ проекционный оператор, принимающий значения 0 на узле занятом железом и 1 - кобальтом $\left(p_{v}^{2}=p_{v}\right), n_{v, \sigma}=a_{v \sigma}^{+} a_{v, \sigma}$, $\delta S_{v}^{(z)}=S_{v}^{(z)}-\sum_{\sigma} \sigma\left\langle n_{\sigma}\right\rangle_{0} / 2, \quad S_{v}^{(z)}=\sum_{\sigma} \sigma n_{v, \sigma} / 2, \quad \delta n_{v}=n_{v}$ $-\sum_{\sigma}\left\langle n_{\sigma}\right\rangle, n_{v}=\sum_{\sigma} n_{v, \sigma},\left\langle n_{v, \sigma}\right\rangle_{0}=\left\langle n_{\sigma}\right\rangle_{0}$ - числа заполнения спиновых $d$-состояний на узле в приближениях $\mathrm{LSDA}+\mathrm{U}+\mathrm{SO}$ и виртуального кристалла.

В силу релятивистской малости ДМ-взаимодействия, ограничимся его учетом в приближении среднего поля, и будем пренебрегать различием параметров межузельного спин-орбитального взаимодействия между узлами оккупированными атомами $3 d$-металлов $(\mathrm{Fe}-\mathrm{Fe}, \mathrm{Co}-\mathrm{Co}$, $\mathrm{Fe}-\mathrm{Co})$

$$
H_{\mathrm{DM}} \approx \sum_{\mathbf{q}= \pm \mathbf{q}_{0}}\left(\mathbf{h}_{-\mathbf{q}}^{(D)} \mathbf{S}_{\mathbf{q}}-\mathbf{d}_{\mathbf{q}}\left[\mathbf{M}_{\mathbf{q}} \times \mathbf{M}_{-\mathbf{q}}\right]\right)
$$

где $\mathbf{d}_{\mathbf{q}}=i d \mathbf{q}, d-$ константа Дзялошинского, $\mathbf{q}-$ вектор квазиимпульса, $\mathbf{M}_{\mathbf{q}_{0}}\left(=\left\langle\mathbf{S}_{\mathbf{q}_{0}}\right\rangle\right)-$ вектор неоднородной намагниченности, $\mathbf{h}_{\mathbf{q}_{0}}^{(D)}=\left[\mathbf{M}_{\mathbf{q}_{0}} \times \mathbf{d}_{-\mathbf{q}_{0}}\right]-$ среднее поле Дзялошинского, $\mathbf{S}_{\mathbf{q}}=\sum_{\sigma, \sigma^{\prime}} a_{\mathbf{k}, \sigma}^{+} \boldsymbol{\sigma}_{\sigma, \sigma^{\prime}} a_{\mathbf{k}+\mathbf{q}, \sigma^{\prime}}^{+}-$Фурьеобраз оператора спиновой плотности на узле, $\boldsymbol{\sigma}_{\sigma, \sigma^{\prime}}$ вектор матриц Паули.

Переходя к определению статистической суммы, воспользуемся мацубаровским представлением взаимодействия $\left(H(\tau)=\exp \left(-\tau H_{0}\right) H \exp \left(\tau H_{0}\right)\right)$,

$$
Z=\mathrm{Sp} T_{\tau} \exp \left(-\int_{0}^{T^{-1}} H(\tau) d \tau\right),
$$

где $T$ - температура в энергетических единицах, $T_{\tau}-$ оператор упорядочения по мацубаровскому времени $\tau$. Для восстановления исходно изотропной записи гамильтониана Хаббарда введем единичный по модулю вектор $\mathbf{e}_{v}$, направленный вдоль оси квантования оператора спина на узле $\boldsymbol{v}$ в момент „времени“ $\tau-\mathbf{S}_{v}=S_{v}^{(z)} \mathbf{e}_{v}$, и выполним усреднение выражения для статистической суммы по всем возможным направлениям этих векторов $[15]$

$$
\begin{aligned}
Z= & \int_{0}^{4 \pi}(d \Omega) \operatorname{Sp} T_{\tau} \exp \left\{-H_{0} / T-\sum_{v}\left(U_{\mathrm{Fe}}-U_{\mathrm{Co}}\right) \delta p_{\boldsymbol{v}}\right. \\
& \times\left[\sum_{\sigma} \sigma\left\langle n_{\sigma}\right\rangle_{0} \mathbf{e}_{v} \delta \mathbf{S}_{v}-\sum_{\delta}\left\langle n_{\sigma}\right\rangle_{0} \delta n_{v} / 2\right] \\
& \left.+\sum\left(U_{\mathrm{Fe}}\left(1-p_{\boldsymbol{v}}\right)+U_{\mathrm{Co}} p_{\boldsymbol{v}}\right)\left[\left(\mathbf{e}_{v} \delta \mathbf{S}_{\boldsymbol{v}}\right)^{2}-\left(\delta n_{v}\right)^{2} / 4\right]\right\},
\end{aligned}
$$

где $(d \Omega)=\prod_{v} d \Omega_{v}, d \Omega_{v}-$ элемент телесного угла направлений единичного вектора $\mathbf{e}_{v}, v=(\boldsymbol{v}, \tau)$, $\sum_{v}(\ldots)=\sum_{v} \int_{0}^{T^{-1}}(\ldots) d \tau$.

Далее, используем процедуру формализма преобразований Стратоновича-Хаббарда [16], сводящих многочастичные взаимодействия в (2) (соответствующие квадратичным слагаемым по оператору спиновой плотности) к взаимодействию электронов с флуктуирующими обменными $(\xi)$ полями. В результате, имеем

$$
\begin{aligned}
Z= & \int(d \xi) \exp \left\{-\sum_{q}\left|\xi_{q}-\mathbf{e}_{0} \delta_{q, 0} c^{-1} \Delta-\delta_{q_{1} \pm \mathbf{q}_{0}} c^{-1} \mathbf{h}_{q}^{(D)}\right|^{2}\right\} \\
& \times Z\left(\xi,\left\langle n_{\sigma}\right\rangle_{0}\right),
\end{aligned}
$$


где $Z\left(\xi,\left\langle n_{\sigma}\right\rangle_{0}\right)=\operatorname{Sp} T_{\tau} \exp \left(-T^{-1} H_{0}^{(L D A)}-T^{-1} \widetilde{\mathscr{H}}\right)$,

$$
\begin{gathered}
H_{0}^{(\mathrm{LDA})}=\sum_{\mathbf{k}, \sigma} \varepsilon_{\mathbf{k}}^{(\mathrm{LDA})} a_{\mathbf{k}, \sigma}^{+} a_{\mathbf{k}, \sigma}, \\
\widetilde{\mathscr{H}}=2 \sum_{q} \mathbf{S}_{q} \xi_{-\mathbf{q}}+i \sum_{q} n_{q} \rho_{-\mathbf{q}}, \\
\xi_{-\mathbf{q}}=\left(c \xi_{-q}-\left(U_{\mathrm{Co}}-U_{\mathrm{Fe}}\right) \sum_{\nu} \delta p_{v} \sum_{\sigma} \sigma \mathbf{e}_{v}\left\langle n_{\sigma}\right\rangle_{0} e^{i q v} / 4\right), \\
\rho_{-\mathbf{q}}=\left(c \eta_{-q}-\left(U_{\mathrm{Co}}-U_{\mathrm{Fe}}\right) \sum_{\nu} \delta p_{v} \sum_{\sigma}\left\langle n_{\sigma}\right\rangle_{0} e^{i q v} / 4\right),
\end{gathered}
$$

$\mathbf{S}_{q}-$ Фурье-образы оператора вектора $\mathbf{S}_{v}, c=(U T)^{1 / 2}$, $U=(1-p) U_{\mathrm{Fe}}+p U_{\mathrm{Co}}, q=\left(\mathbf{q}, \omega_{2 n}\right), \omega_{2 n}-$ мацубаровская Бозе-частота.

Поскольку в модели хаотического сплава зарядовое упорядочение невозможно, а флуктуации зарядовой плотности ведут к большим флуктуациям энергии и, следовательно, маловероятны, постольку при расчете $Z\left(\xi\left\langle n_{\sigma}\right\rangle_{0}\right)$ слагаемыми, пропорциональными $\rho_{q}$ с $q \neq 0$, в эффективном гамильтониане $\widetilde{\mathscr{H}}$ можно пренебречь. В результате эффективный гамильтониан $\widetilde{\mathscr{H}}$ переписывается к виду

$$
\widetilde{\mathscr{H}}=2 \sum_{q} \mathbf{S}_{q} \xi_{-q}+i n_{0} \rho_{0}
$$

Квантово-статистическое вычисление выражения для функционала свободной энергии $Z\left(\xi\left\langle n_{\sigma}\right\rangle_{0}\right)$, рассматриваемой задачи о фазовых переходах в киральных магнетиках с аномально большими периодами магнитной спиновой структуры, выполним на основе приближения однородных локальных полей [17]. Разлагая (5) по степеням $\widetilde{\mathscr{H}}$ и проводя квантовостатистическое усреднение, получаем ряд по степеням внутренних обменных и зарядовых полей, действующих на электроны, $-\boldsymbol{\xi}_{-q}$ и $\rho_{0}$. Вершинные части ряда выражаются через функции Грина в приближениях LDA + U + SO (описываемом гамильтонианом $\left.H_{0}^{(\mathrm{LDA})}\right)$ и виртуального кристалла: $\varphi^{(l)}\left(q_{1} \ldots q_{l}\right)=$ $=T \sum_{k} G_{k+q_{1}}^{(0)} G_{k+q_{1}+q_{2}}^{(0)} \ldots G_{k+\sum^{\prime} g_{i}}^{(0)} \sum^{l} q_{i ; 0}$. В приближении однородных локальных полей они аппроксимируются выражением: $\varphi^{(l)}\left(q_{1} \ldots q_{l}\right)=\delta_{\sum^{l} q_{i ; 0}} \varphi^{(l)}(0, \ldots, 0)$ $\times \prod_{i}^{l} \theta\left(q_{i}-q_{C}\right)$. Однако слагаемые, соответствующие вершинным частям второго порядка, определяют $(\mathbf{q}, \omega)$-зависимость обратного значения аномально в окрестности фазового перехода фактора обменного усиления $D$. Поэтому во втором порядке пренебрегать пространственно-временной неоднородностью нельзя. Последняя описывается фактором обменного усиления, аномально сильно зависящим от $\mathbf{q}$ и $\omega$ в ферромагнитных и длиннопериодических спиновых структурах. В рамках (см., например, [18]) известной аппроксимации функции Линдхарда $\left(\chi^{(0)}(\mathbf{q}, \omega)\right)$, имеем

$$
\begin{aligned}
\chi^{(0)}(\mathbf{q}, \omega)= & \chi^{(0)}(0,0)+\chi^{(0)}(0,0)\left(A\left(\mathbf{q} / k_{F}\right)^{2}-i B U^{-1}\right. \\
& \left.\times \frac{\omega}{\left|\mathbf{q} / k_{F}\right|} \theta\left(T_{0}\left|\mathbf{q} / k_{F}\right|-\omega\right)\right) \theta\left(2 k_{F}-|\mathbf{q}|\right),
\end{aligned}
$$

где $T_{0}=V_{F} k_{F}, V_{V}-$ скорость на поверхности Ферми, $k_{F}-$ модуль вектора Ферми, $\theta(x)-\theta$-функция, коэффициенты $A$ и $B$ пропорциональны плотности электронных состояний на уровне Ферми и в случае $3 d$-переходных металлов и их соединений, имеют значения порядка 0.1 и 1 [18] соответственно.

Вычисления функциональных интегралов выполним в приближении метода наибыстрейшего спуска по переменным: $\xi_{0}, \quad \xi_{ \pm q_{0}}, \quad \rho_{0}, \quad\left|\xi_{q}^{(\gamma)}\right|$ и $\varphi_{q}^{(\gamma)}\left(=\arg \xi_{q}^{(\gamma)}\right)$, перевальные значения которых определяются условиями максимума подынтегрального выражения в (4).

Анализ выражения для статистической суммы (4) показывает, что имеется связь между перевальными значениями $\xi-$ полей с намагниченностями $M_{0}=$ $=U^{-1}\left(c \xi_{0}^{(z)}-U \sum_{\sigma} \sigma\left\langle n_{\sigma}\right\rangle_{0} / 2\right)$ и $\mathbf{M}_{\mathbf{q}_{0}, m}=U^{-1}\left(c \xi_{\mathbf{q}_{0}}-\mathbf{h}_{\mathbf{q}_{0}}\right)$ на векторах $\mathbf{q}=0$ и $\pm \mathbf{q}_{0}$ соответственно, а также со спиновой мацубаровской функцией Грина $\left\langle T_{\tau} \mathbf{S}_{q} \mathbf{S}_{-q}\right\rangle=(c / U)^{2}\left(2\left|\boldsymbol{\xi}_{q}\right|^{2}-1\right)$ и среднеквадратическим магнитным моментом на узле, определяемом значениями модуля намагниченности и амплитуды спиновых флуктуаций

$$
\begin{gathered}
m^{2} \approx\left|\mathbf{M}_{\mathbf{q}_{0}}\right|^{2}+\left\langle m^{2}\right\rangle \\
\left.\left\langle m^{2}\right\rangle=(2 \pi)^{-1} \sum_{\mathbf{q}\left(\neq \mathbf{q}_{0}\right)} \int_{0}^{\infty}\left(1+2 f_{B}(\omega / T)\right) \operatorname{Im} / T_{\tau} \mathbf{S}_{q, m} \mathbf{S}_{-q, m}\right\rangle d \omega \\
+\frac{\left(U_{\mathrm{Co}}-U_{\mathrm{Fe}}\right)^{2}}{4 U^{2}}\left(\sum_{\sigma} \sigma\left\langle n_{\sigma}\right\rangle_{0}\right)^{2} N_{0}^{-1} \sum_{\nu} \delta p_{v}^{2}
\end{gathered}
$$

где $f_{B}(\omega / T)-$ функция Бозе.

Перевальные значение $\rho_{q}=0$, а $\rho_{0}$ определяется условием

$$
c \rho_{0}=2^{-1} U\left(n-\sum_{\sigma}\left\langle n_{\sigma}\right\rangle_{0}\right),
$$

где $n$ - заполнение $t_{0}$-зоны, плотность электронных состояний в которой перенормирована флуктуациями электронной спиновой плотности

$$
\begin{gathered}
g(\varepsilon)=2^{-1} \sum_{\alpha= \pm 1} g_{a}(\varepsilon), \\
g_{\alpha}(\varepsilon)=2^{-1} \sum_{\sigma} g_{\sigma}^{(\mathrm{LSDA})}\left(\varepsilon-c \rho_{0}+\sigma \Delta+\alpha U m\right) .
\end{gathered}
$$

При этом химический потенциал $(\mu)$ определяется исходя из условия

$$
x=\int f(\varepsilon-\mu) g(\varepsilon) d \varepsilon,
$$

$f(\varepsilon-\mu)-$ функция Ферми-Дирака. 


\section{3. Уравнение магнитного состояния}

Уравнения магнитного состояния, получаемые из условий минимума (4) по однородной и неоднородной намагниченности, содержат эффекты, связанные с магнитными флуктуациями

$$
\begin{aligned}
\mathbf{M}_{\mathbf{q}_{0}}\left(D^{-1}+X\left(\mathbf{q}_{0}, 0\right)\right)+\kappa \mathbf{M}_{-\mathbf{q}_{0}}\left(\mathbf{M}_{\mathbf{q}_{0}}\right)^{2} & \\
= & \left(1-D^{-1}-X\left(\mathbf{q}_{0}, 0\right)\right) \mathbf{h}_{\mathbf{q}_{0}}^{(D)}, \\
M_{0} D^{-1}= & -\Delta\left(D^{-1}-\kappa\left(\left\langle m^{2}\right\rangle\right) / 3\right),
\end{aligned}
$$

где $\quad \mathbf{M}_{\mathbf{q}_{0}}\left(=\mathbf{M}_{-\mathbf{q}_{0}}^{*}\right)=2^{-1 / 2}\left(\mathbf{i} M_{\mathbf{q}_{0}}^{(x)}+\mathbf{j} M_{\mathbf{q}_{0}}^{(y)}\right), \quad \mathbf{i}$ и $\quad \mathbf{j}-$ орты, лежащие в геликоидальной плоскости, $D^{-1}=1-U \chi_{\perp}+\kappa\left(\left\langle m^{2}\right\rangle\right) / 3-$ фактор обменного усиления однородной магнитной восприимчивости, $\kappa\left(U / m^{2}\right)\left\lfloor\chi_{\perp}-\chi_{\|}\right\rfloor-$коэффициент межмодовой связи, $\chi_{\perp}=(2 U m)^{-1} \Delta n$ и $\chi_{\|}=2\left(\sum_{\alpha= \pm 1} g_{\alpha}(\mu)\right)^{-1} \prod_{\alpha= \pm 1} g_{\alpha}(\mu)$, поперечная и продольная восприимчивости соответственно, $\quad \Delta n=\sum_{\alpha= \pm 1} \alpha \int_{-\infty}^{\infty} g_{\alpha}(\varepsilon) f(\varepsilon-\mu) d \varepsilon-$ разность чисел заполнения состояний с $\alpha= \pm 1$.

Известная из LSDA + U + SO-расчетов спиновая система является ферромагнитной, а электронная структура представляет собой расщепленные по направлениям спина спин-поляризованные подзоны с раздвижкой пропорциональной намагниченности $[10,19]$. Однако учет ДМ-взаимодействия „закручивает“ спины, и однородная намагниченность исчезает. Анализ $(5,8,9)$ показывает, что в этом случае $M_{0}=0$ и раздвижка спинполяризованных подзон отсутствует, хотя в исходной LSDA-модели $\Delta \neq 0$. При этом согласно (9) в основном состоянии $(T=0)$ возникает среднее значение модуля локального магнитного момента

$$
\left(\left|M_{\mathbf{q}_{0}}\right|^{2}+\left\langle m^{2}\right\rangle\right)^{1 / 2}=2^{-1} \sum_{\alpha= \pm 1} \alpha \int_{-\infty}^{\infty} g_{\alpha}(\varepsilon) f(\varepsilon-\mu) d \varepsilon,
$$

который при малых значениях $\mathbf{M}_{\mathbf{q} 0}\left(\sim \mathbf{h}_{-\mathbf{q}_{0}}^{(D)}\right)$, связан с флуктуациями магнитных моментов на узлах занятых железом и кобальтом (6). Наличие таковых позволяет согласовать экспериментальные данные по магнитным моментам с результатами наших расчетов (рис. 1).

При этом, несмотря на ненулевые значения $\left\langle n_{\sigma}\right\rangle_{0}$, в рассматриваемом случае ферромагнитного геликоида отсутствует остаточная однородная намагниченность, а однородная магнитная восприимчивость, которую можно получить дифференцируя (8), имеет вид

$$
\chi=2 U^{-1}\left[\left(X\left(\mathbf{q}_{0}, 0\right)+\kappa\left|\mathbf{M}_{\mathbf{q}_{0}}\right|^{2}\right)^{-1}-1\right] .
$$

Как показывают численные расчеты величина амплитуды спиновых флуктуаций (7) в приближении хаотиче-

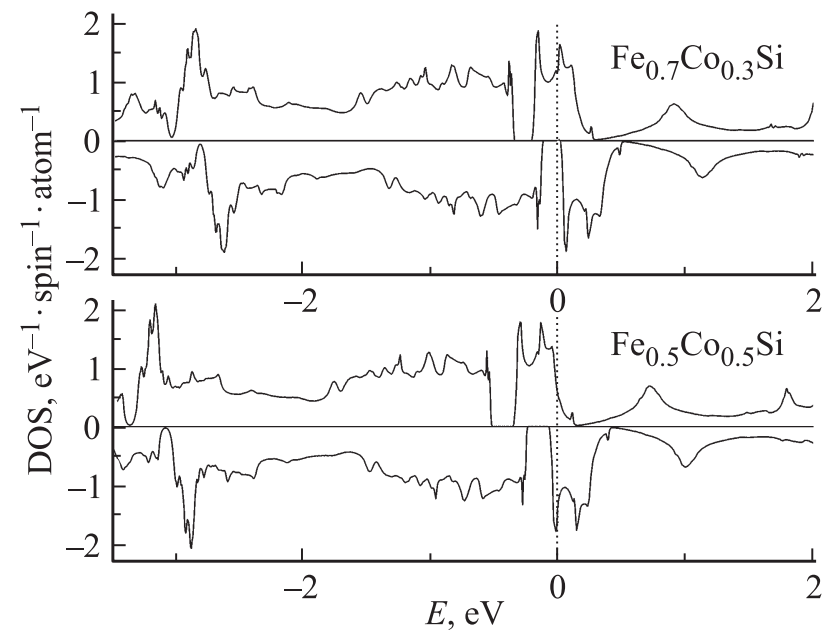

Рис. 1. Плотность электронных состояний для основного состояния $\mathrm{Fe}_{1-x} \mathrm{Co}_{x} \mathrm{Si}$ в LSDA $+\mathrm{U}+\mathrm{SO}$. Положение химического потенциала совпадает с началом отсчета энергии. Параметры первопринципного моделирования электронной структуры: величина хаббардовского отталкивания $U=0.78$ и $0.795 \mathrm{eV}$ для сплавов $\mathrm{Fe}_{0.3} \mathrm{Co}_{0.7} \mathrm{Si}$ и $\mathrm{Fe}_{0.5} \mathrm{Co}_{0.5} \mathrm{Si}$ соответственно; параметр хундовского обменного взаимодействия $J=0.428$ и $0.435 \mathrm{eV}$, для сплавов $\mathrm{Fe}_{1-x} \mathrm{Co}_{x} \mathrm{Si}$ с содержанием кобальта $x=0.3,0.5$ соответственно.

ского сплава аппроксимируется выражением

$$
\begin{aligned}
& \left\langle m^{2}\right\rangle=(3 / 4) B(T / U)^{2}\left(D^{-1}+2 \kappa M_{S}^{2}\right)^{-1} \\
& \times\left(D^{-1}+2 \kappa M_{S}^{2}+A\right)^{-1}+\frac{\left(U_{\mathrm{Co}}-U_{\mathrm{Fe}}\right)^{2}}{4 U^{2}} \sum_{\sigma} \sigma\left\langle n_{\sigma}\right\rangle_{0} x(1-x) .
\end{aligned}
$$

\section{4. Решения, соответствующие модели Янсена-Бака $\left(T<T_{C}\right)$}

Решения уравнения (8) при $\kappa>0$ соответствуют ферромагнитному геликоиду с фиксированным вектором амплитуды геликоидальной структуры. В таком киральном магнетике

$$
M_{\mathbf{q}_{0}}^{(x)}=M_{S}, \quad M_{\mathbf{q}_{0}}^{(y)}=-i M_{S}, \quad N_{0}^{-1} \sum_{\nu} M_{\nu}^{(\gamma)}=M_{S},
$$

где $M_{S}=(2 \kappa)^{-1 / 2}\left(\left(D^{-1}+X\left(\mathbf{q}_{0}, 0\right)\right)^{2}-\left(d q_{0} / U\right)^{2}\right)^{1 / 4}$, а волновой вектор гелимагнитного упорядочения q определяется условием максимума модуля вектора амплитуды неоднородной намагниченности и удовлетворяет равенству $\left|\mathbf{q}_{0}\right| \approx d / 2 U A$. При этом направление вектора $\mathbf{q}_{0}$ и вращения спинов определяются знаком параметра DМ-взаимодействия.

Результаты расчета неоднородной намагниченности представлены на рис. 2. Там же представлены результаты расчета однородной магнитной восприимчивости 


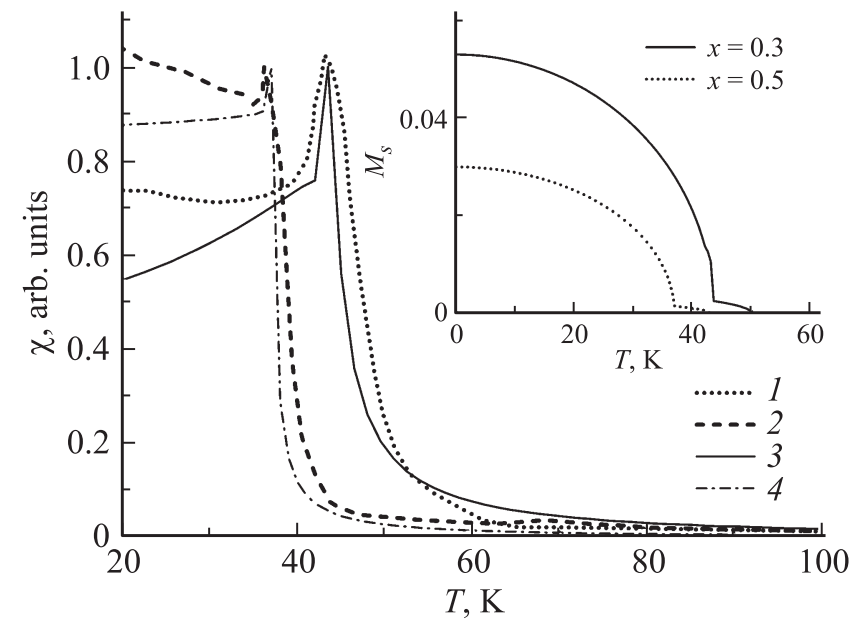

Рис. 2. Температурная зависимость однородной магнитной восприимчивости соединений $\mathrm{Fe}_{1-x} \mathrm{Co}_{x} \mathrm{Si}: 1,2$ - экспериментальные данные для $\mathrm{Fe}_{0.7} \mathrm{Co}_{0.3} \mathrm{Si}$ [9] и $\mathrm{Fe}_{0.5} \mathrm{Co}_{0.5} \mathrm{Si}$ [4] соответственно; 3,4- расчет в настоящей работе по формуле (12) для $\mathrm{Fe}_{0.7} \mathrm{Co}_{0.3} \mathrm{Si}$ и $\mathrm{Fe}_{0.5} \mathrm{Co}_{0.5} \mathrm{Si}$ соответственно. На вставке: температурная зависимость модуля амплитуды спиновой плотности для $\mathrm{Fe}_{1-x} \mathrm{Co}_{x} \mathrm{Si}(x=0.3,0.5)$.

по формуле (13), которые хорошо описывают экспериментально наблюдаемую зависимость $\chi(T)$ вблизи температуры $T_{C}$ (рис. 2).

\section{5. Киральные флуктуации $\left(T_{C}<T<T_{S}\right)$}

В условиях, когда $\kappa<0$, геликоидальное ферромагнитное состояние с фиксированной по направлению осью квантования и вектором неоднородной намагниченности $\mathbf{M}_{\mathbf{q}_{0}}$ исчезает. При этом сохраняются ферромагнитные спиновые корреляции, радиус которых определяется выражением

$$
R_{C}=k_{F}^{-1} A^{1 / 2}\left(X\left(\mathbf{q}_{0}, 0\right)+\kappa\left|\mathbf{M}_{\mathbf{q}_{0}}\right|^{2}\right)^{-1 / 2} \sim(U \chi)^{1 / 2} .
$$

Согласно развиваемой численной модели при температуре $T_{C}$ радиус спиновых корреляций равен периоду геликоидальной спирали, а при $T>T_{C}$ убывает с возрастанием температуры (рис. 3).

В результате возникает магнитное состояние с $\kappa<0$ и $D^{-1}>0$, причем

$$
M_{v}^{(x)}=M_{S} \cos \left(\mathbf{q}_{0} v+\phi\right) \quad \text { и } \quad M_{v}^{(y)}=-M_{S} \sin \left(\mathbf{q}_{0} v+\phi\right),
$$

где фаза $\phi$ меняется стохастически (вследствие исчезновения ферромагнитной оси квантования). Последнее означает, что

$$
N_{0}^{-1} \sum_{\nu} M_{\nu}^{(\gamma)}=(2 \pi) \int_{0}^{2 \pi} M_{\nu}^{(\gamma)} d \varphi=0,
$$

и отвечает возникновению киральных флуктуаций вектора спиновой плотности $\mathbf{M}_{v}$,

$$
N_{0}^{-1} \sum_{\nu}\left\langle S_{v}^{(\gamma)}\right\rangle^{2}=(2 \pi)^{-1} \int_{0}^{2 \pi} M_{v}^{(\gamma)^{2}} d \phi=M_{S}^{2} .
$$

Решение уравнения магнитного состояния допускает возникновение фиксированных значений фазы в пределах радиуса корреляций. При условиях флуктуаций стохастических фаз за его пределами.

Модуль амплитуды спиновой плотности на векторе геликоидального упорядочения

$$
\begin{gathered}
M_{S}=\left\langle m^{2}\right\rangle_{T_{C}}^{1 / 2}\left(1-\left(T / T_{S}\right)^{2}\right)^{1 / 2} \\
T_{S}=|\kappa|^{-1} B^{-1} U\left(\left|D\left(\left\langle m^{2}\right\rangle_{T_{C}}^{1 / 2}\right)^{-1}\right|-d q_{0} / U\right)^{1 / 2} .
\end{gathered}
$$

При этом отличие температур $T_{C}$ и $T_{S}$ оказывается обусловленным различием параметров хаббардовского взаимодействия на узлах, занятых атомами $\mathrm{Fe}$ и $\mathrm{Co}$,

$$
T_{S}^{2}=T_{C}^{2}+\left(\frac{U_{\mathrm{Co}}-U_{\mathrm{Fe}}}{2 U} \sum_{\sigma} \sigma\left\langle n_{\sigma}\right\rangle_{0}\right)^{2} x(1-x) .
$$

Отличие потенциалов железа и кобальта от потенциала виртуального кристалла $U$ приводит к локальной намагниченности выше $T_{C}$. При значении температуры равном $T_{S}$ локальная намагниченность исчезает, причем расчетное значение радиуса корреляций примерно равно $0.6 \lambda$ (согласно данным малоуглового рассеяния нейтронов $-0.7 \lambda)$, что отвечает флуктуационному ближнему порядку.

Таким образом, рассматриваемый магнитный переход является затянутым по температуре. При температуpe $T_{C}$ исчезает фиксированное направление ферромагнитной оси квантования, а при $T_{S}$ исчезает локальная намагниченность.

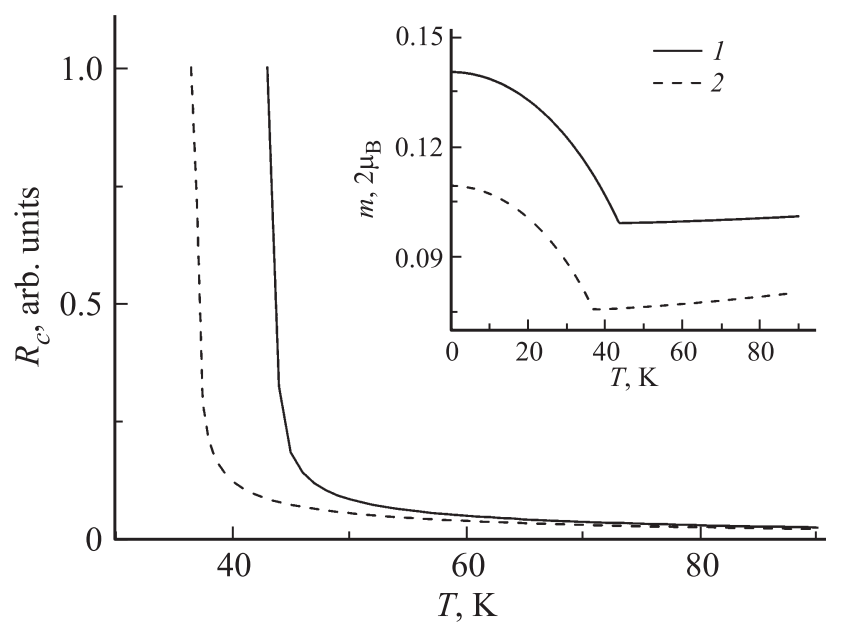

Рис. 3. Температурная зависимость радиуса корреляции, рассчитанного в настоящей работе; на вставке: температурная зависимость локального магнитного момента для сплавов $\mathrm{Fe}_{1-x} \mathrm{Co}_{x} \mathrm{Si}: 1-x=0.3,2-x=0.5$. 


\section{6. Теплоемкость и тепловое расширение}

Вблизи температур магнитного перехода $T_{C}$ и $T_{S}$ электронные и магнитные вклады в свободную энергию, согласно формулам (4)-(7) перенормированы за счет расщепления электронных спектров во флуктуирующих обменных полях

$$
F=-T \ln Z=F_{\mathrm{el}}+F_{\mathrm{mag}},
$$

где

$$
\begin{aligned}
R_{\mathrm{el}} & =T \sum_{m} \int_{-\infty}^{\infty} g(\varepsilon) \ln (1-f(\varepsilon-\mu)) d \varepsilon-\mu N \\
F_{\mathrm{mag}}= & \sum_{q}\left(1+X_{q}\right)\left|M_{q}\right|^{2}+\sum_{\gamma}\left(D^{-1}+2 \kappa\left|M_{\mathbf{q}_{0}}^{(\gamma)}\right|\right)\left\langle m^{2}\right\rangle_{T} \\
& +(2 \pi)^{-1} \sum_{\mathbf{q}\left(\neq \mathbf{q}_{0}\right), \gamma} \int_{0}^{\infty}\left(2 f_{B}(\Omega / T)\right) \\
& \times \operatorname{Im}\left[\ln \left(D^{-1}+2 \kappa\left|M_{\mathbf{q}_{0}}^{(\gamma)}\right|+X_{\mathbf{q}}(\omega)\right)\right] d \omega .
\end{aligned}
$$

В соответствии с термодинамическим определением $\left(C_{V}=T\left(\partial^{2} T \ln Z / \partial T^{2}\right)\right)_{V}$ электронный вклад в теплоемкость при постоянном объеме определяется выражением

$$
\begin{aligned}
C_{V}= & \frac{\pi^{2}}{3} g(\mu) T+U\left(D^{-1}+A(a / \lambda)^{2}+A\right) \frac{d\left\langle m^{2}\right\rangle}{d T} \\
& -U \kappa\left(M_{S}^{2}+\left\langle m^{2}\right\rangle / 3\right) \frac{d m^{2}}{d T} .
\end{aligned}
$$

Поскольку в условиях термодинамической неустойчивости ферромагнитного упорядочения $\left(T_{C}<T<T_{S}\right)$ и температурной ее окрестности значения коэффициента межмодовой связи малы $\left(\kappa\left(T_{C}\right)=0\right.$ и $\left.\kappa\left(T_{S}\right) \approx 0^{1}\right)$, a $\left|D^{-1}+A(a / \lambda)^{2}\right| \sim d / U \ll A$, постольку электронный вклад в теплоемкость не проявляет аномалий в точках $T_{C}$ и $T_{S}$ (см. вставка на рис. 4) $-C_{V} \approx U A d\left\langle m^{2}\right\rangle / d T$.

Учет ангармонизма, связанного с тепловым расширением, в выражении для теплоемкости при постоянном давлении (см. вставка к рис. 4)

$$
C_{p}=C_{V}+K \beta(T)(V(T)-V(0)) / V(0)
$$

здесь не рассматривается, а на эксперименте не проявляется, вследствие того, что относительное изменение объема является не только малым, но и непрерывным. Поэтому в эксперименте $[4,6]$ какая-либо корреляция зависимостей $C(T)$ и $\beta(T)$ отсутствует.

Для определения ОКТР необходимо рассмотреть магнитообъемный эффект. Давление, возникающее при изменении объема,

$$
P=K \omega=-(\partial F / \partial V)_{T}, \quad \omega=\Delta V / V,
$$

\footnotetext{
${ }^{1}$ В рассматриваемой численной модели плотности электронных состояний, повторное обращение в нуль коэффициента межмодовой связи $\kappa$ имеет место при температуре больше $T_{S}$ на $5 \mathrm{~K}$.
}

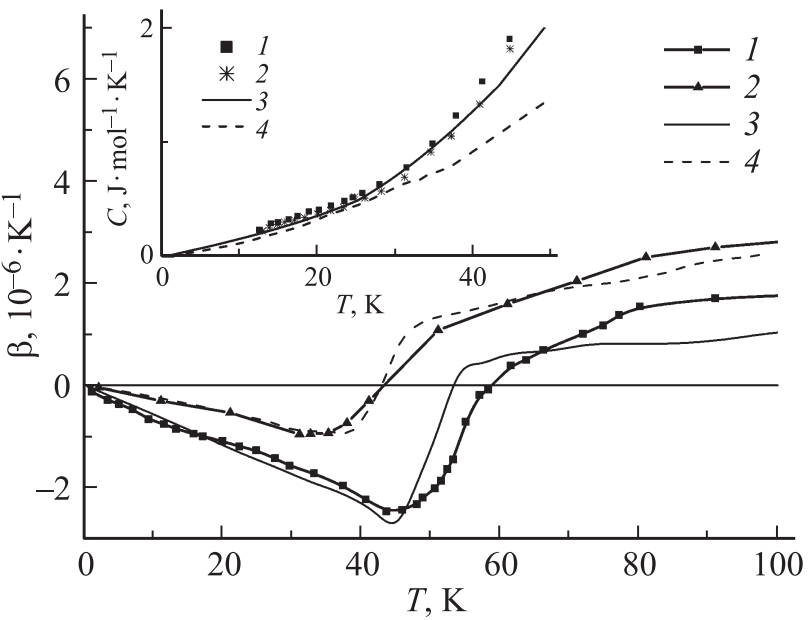

Рис. 4. Температурная зависимость объемного коэффициента теплового расширения сплавов $\mathrm{Fe}_{1-x} \mathrm{Co}_{x} \mathrm{Si}: 1,2$ - экспериментальные данные для $\mathrm{Fe}_{0.7} \mathrm{Co}_{0.3} \mathrm{Si}$ и $\mathrm{Fe}_{0.5} \mathrm{Co}_{0.5} \mathrm{Si}$ [6] соответственно; 3,4- расчет в настоящей работе для $\mathrm{Fe}_{0.7} \mathrm{Co}_{0.3} \mathrm{Si}$ и $\mathrm{Fe}_{0.5} \mathrm{Co}_{0.5} \mathrm{Si}$ соответственно. На вставке - температурная зависимость теплоемкости соединений $\mathrm{Fe}_{1-x} \mathrm{Co}_{x} \mathrm{Si}: 1,2-$ экспериментальные данные для соединений $\mathrm{Fe}_{0.7} \mathrm{Co}_{0.3} \mathrm{Si}$ и $\mathrm{Fe}_{0.5} \mathrm{Co}_{0.5} \mathrm{Si}[20]$ соответственно; $3,4-$ расчет в настоящей работе для $\mathrm{Fe}_{0.7} \mathrm{Co}_{0.3} \mathrm{Si}$ и $\mathrm{Fe}_{0.5} \mathrm{Co}_{0.5} \mathrm{Si}$.

может быть определено в модели соответственных состояний [21], согласно которой плотность электронных состояний

$$
\begin{aligned}
g_{a}(\varepsilon)= & 2^{-1}\left(W / W_{0}\right)^{-1} \\
& \times \sum_{\sigma} g_{\sigma}^{(\mathrm{LSDA})}\left(\varepsilon-c \rho_{0}+\sigma \Delta+\alpha U m W_{0} / W\right),
\end{aligned}
$$

где относительная ширина зоны $W / W_{0} \sim\left(V / V_{0}\right)^{-n / 3}$ $\left(n=5\right.$ для $d$-электронов), $V_{0}$ и $W_{0}$ - объем кристалла и ширина зоны в отсутствии теплового расширения, $K-$ коэффициент изотермической сжимаемости.

Тогда, используя термодинамическое условие минимума свободной энергии для относительного изменения объема и условия перевала, находим ОКТР $(\beta=\partial \omega / \partial T)$

$$
\begin{aligned}
\beta= & \left(K^{-1} d \ln W / d \ln V\right)\left[2 \frac{\pi^{2}}{3} g(\mu) T+U\left(2 \kappa \frac{d M_{S}^{2}}{d T}\left\langle m^{2}\right\rangle\right.\right. \\
& \left.\left.+A(a / \lambda)^{2} \frac{d\left\langle m^{2}\right\rangle}{d T}+\left(\kappa m^{2}+2 U \tilde{g}(\mu)\right)\left(\frac{d m^{2}}{d T}\right)\right)\right],
\end{aligned}
$$

в котором содержится магнитный вклад, величина которого определяется первыми производными по температуре от локальной намагниченности, амплитуды спиновых флуктуаций и среднеквадратического спинового магнитного момента. При этом величина ОКТР является отрицательной при $T<T_{S}$ и меняет знак при переходе из киральной в парамагнитную вследствие исчезновения модуля локальной намагниченности на узле. 
Изменение знака коэффициента межмодовой связи $\kappa$ в точке $T_{C}$ приводит к формированию экстремума на зависимости $\beta(T),{ }^{2}$ который также как и смена знака этой зависимости в точке $T_{C}$ представлены на рис. 4 и согласуются с экспериментальными данными. Отметим, что магнитный вклад оказывается значительно больше вклада фермиевских возбуждений (согласно (19) в $U / T$-раз).

\section{7. Заключение}

В настоящей работе показано, что магнитный переход в геликоидальных ферромагнетиках $\mathrm{Fe}_{1-x} \mathrm{Co}_{x} \mathrm{Si}$ оказывается затянутым по температуре. Это связано с возникновением киральных флуктуаций, вследствие исчезновения фиксированной ферромагнитной оси квантования (катастрофа модели Бака-Янсена). При этом локальная намагниченность на узле не исчезает, что становится возможным вследствие различия кулоновских потенциалов $\mathrm{Fe}$ и $\mathrm{Co}$ на узлах. Похожая, но иная по природе ситуация, имеет место в $\mathrm{MnSi}$, где ферромагнитная ось квантования исчезает вследствие „скачка“ амплитуды нулевых спиновых флуктуаций [13]. При температуре $T_{S}$ локальная намагниченность исчезает вследствие термодинамических спиновых флуктуаций. При температуре $T_{C}$ в $\mathrm{Fe}_{1-x} \mathrm{Co}_{x} \mathrm{Si}$ (в отличие от $\mathrm{MnSi}[5])$ температурные скачки теплоемкости не имеют место, поскольку спиновые флуктуации, связанные со стохастическими флуктуациями кулоновских потенциалов $\mathrm{Fe}$ и Со не изменяются с температурой. Магнитная восприимчивость и ОКТР $\mathrm{Fe}_{1-x} \mathrm{Co}_{x} \mathrm{Si}$ в возникновении киральных флуктуаций (вблизи $T_{C}$ ) обнаруживают температурные экстремумы, связанные с изменением знака коэффициента межмодовой связи $\kappa$, который определяет устойчивость ферромагнитных решений в уравнении магнитного состояния (9)-(10).

Фазовый магнитный переход в $\mathrm{Fe}_{1-x} \mathrm{Co}_{x} \mathrm{Si}$ оказывается затянутым по температуре и завершается исчезновением при температуре $T_{S}\left(>T_{C}\right)$ локальной намагниченности и киральных флуктуаций. Последнее сопровождается изменением знака ОКТР, поскольку в парамагнитной области сохраняются термодинамические спиновые флуктуации (температурно-индуцированные локальные магнитные моменты), амплитуда которых растет с температурой.

\section{Список литературы}

[1] J. Beille, J. Voiront, F. Towfiq, M. Roth, Z.Y. Zhang. J. Phys. F 11, 2153 (1981).

[2] P. Bak, M.H. Jensen. J. Phys. C 13, L881 (1980).

[3] С.В. Григорьев, В.А. Дядькин, С.В. Малеев, D. Menzel, J. Schoenes, D. Lamago, E.B. Москвин, H. Eckerlebe. ФТT 52, 852 (2010).

\footnotetext{
2 В пренебрежение различием потенциалов хаббардовского взаимодействия железа и кобальта на зависимости $\beta(T)$ возникает скачок при $T=T_{C}$.
}

[4] A. Bauer, M. Garst, C. Pfleiderer. Phys. Rev. B 93, 235144 (2016).

[5] S.M. Stishov, A.E. Petrova, S. Khasanov, G.Kh. Panova, A.A. Shikov, J.C. Lashley, D. Wu, T.A. Lograsso. JETP 106, 888 (2008).

[6] П.В. Гельд, А.А. Повзнер, С.В. Кортов, Р.П. Кренцис. ДАН CCCP 297, 1359 (1987).

[7] V.A. Dyadkin, S.V. Grigoriev, E.V. Moskvin, S.V. Maleyev, D. Menzel, J. Schoenes, H. Eckerlebe. Physica B 404, 2520 (2009).

[8] L.J. Bannenberg, K. Kakurai, P. Falus, E. Lelièvre-Berna, R. Dalgliesh, C.D. Dewhurst, F. Qian, Y. Onose, Y. Endoh, Y. Tokura, C. Pappas. Phys. Rev. B 95, 144433 (2017).

[9] T.Y. Ou-Yang, G.J. Shu, C.D. Hu, F.C. Chou. J. Appl. Phys. 117, 123903 (2015).

[10] X.Z. Yu, N. Kanazawa, Y. Onose, K. Kimoto, W.Z. Zhang, S. Ishiwata, Y. Matsui, Y. Tokura. Nature Mater. 10, 106 (2011).

[11] V.V. Mazurenko, A.O. Shorikov, A.V. Lukoyanov, K. Kharlov, E. Gorelov, A.I. Lichtenstein, V.I. Anisimov. Phys. Rev. B 81, 125131 (2010).

[12] K.V. Shanavas, S. Satpathy. Phys. Rev. B 93, 195101 (2016).

[13] А.А. Повзнер, А.Г. Волков, Т.А. Ноговицына. ФТТ 59, 1261 (2017).

[14] M.M. Steiner, R.C. Albers, L.J. Sham. Phys. Rev. B 45, 13272 (2016).

[15] Т. Мория. УФН 135, 117 (1981).

[16] J. Hubbard. Proc. Roy. Soc. A 276, 238 (1963).

[17] J.A. Hertz, M.A. Klenin. Phys. Rev. B 10, 1084 (1974).

[18] I.E. Dzyaloshinskii, P.S. Kondratenko. Sov. Phys. JETP 43, 1036 (1976).

[19] Hu Zhi-Hui, He Wei, Sun Young, Cheng Zhao-Hua. Chinese Phys. 16, 3863 (2007).

[20] П.В. Гельд, А.А. Повзнер, С.В. Кортов, В.Н. Сафонов. ДАН CCCP 289, 351 (1986).

[21] V. Heine. Phys. Rev. 153, 673 (1967). 